\title{
Weak orderability of second countable spaces
}

\author{
by \\ Valentin Gutev (Durban) \\ Dedicated to the memory of Steve Purisch
}

\begin{abstract}
We demonstrate that a second countable space is weakly orderable if and only if it has a continuous weak selection. This provides a partial positive answer to a question of van Mill and Wattel.
\end{abstract}

1. Introduction. For a $T_{1}$-space $X$, let $\mathscr{F}(X)$ be the set of all nonempty closed subsets of $X$. Usually, we endow $\mathscr{F}(X)$ with the Vietoris topology $\tau_{\mathrm{V}}$, and call it the Vietoris hyperspace of $X$. Recall that $\tau_{\mathrm{V}}$ is generated by all collections of the form

$$
\langle\mathscr{V}\rangle=\{S \in \mathscr{F}(X): S \subset \bigcup \mathscr{V} \text { and } S \cap V \neq \emptyset \text { whenever } V \in \mathscr{V}\},
$$

where $\mathscr{V}$ runs over the finite families of open subsets of $X$.

In the following, all spaces are assumed to be at least Hausdorff, while any subset $\mathscr{D} \subset \mathscr{F}(X)$ will carry the relative Vietoris topology $\tau_{\mathrm{V}}$ as a subspace of the hyperspace $\left(\mathscr{F}(X), \tau_{\mathrm{V}}\right)$. A map $f: \mathscr{D} \rightarrow X$ is a selection for $\mathscr{D}$ if $f(S) \in S$ for every $S \in \mathscr{D}$. A selection $f: \mathscr{D} \rightarrow X$ is continuous if it is continuous with respect to the relative Vietoris topology $\tau_{\mathrm{V}}$ on $\mathscr{D}$.

In this paper, we are especially interested in continuous selections for $\mathscr{D}$ when $\mathscr{D}$ is the set $\mathscr{F}_{2}(X)=\{S \in \mathscr{F}(X):|S| \leq 2\}$ of all nonempty at most 2-point subsets of $X$. Every selection $f: \mathscr{F}_{2}(X) \rightarrow X$ defines a natural order-like relation $\preceq_{f}$ on $X[10]$ by letting $x \preceq_{f} y$ if and only if $f(\{x, y\})=x$. For convenience, we write $x \prec_{f} y$ if $x \preceq_{f} y$ and $x \neq y$. This relation is similar to a linear order on $X$ in that it is both total and antisymmetric, but, unfortunately, it may fail to be transitive. In this connection, one of the fundamental questions in the theory of continuous selections for at most 2-point subsets is the following.

2000 Mathematics Subject Classification: 54B20, 54C65, 54F05.

Key words and phrases: Vietoris hyperspace, continuous selection, weak selection, weakly orderable space.

This work is based upon research supported by the NRF of South Africa. 
Question 1 (van Mill and Wattel, [11]). Let $X$ be a space which has a continuous selection for $\mathscr{F}_{2}(X)$. Does there exist a linear order $\preceq$ on $X$ such that, for each $y \in X$, the sets $\{x \in X: x \preceq y\}$ and $\{x \in X: y \preceq x\}$ are both closed?

Recall that a space $X$ is orderable (or linearly orderable) if the topology of $X$ coincides with the open interval topology on $X$ generated by a linear ordering on $X$. Following [11], we say that a space $X$ is weakly orderable if there exists a coarser orderable topology on $X$. In this terminology, Question 1 asks if a space $X$ is weakly orderable provided it has a continuous selection for $\mathscr{F}_{2}(X)$. In view of that, a selection $f: \mathscr{F}_{2}(X) \rightarrow X$ is often called a weak selection for $X$. For a detailed discussion of Question 1, we refer the interested reader to [7].

The purpose of this paper is to prove the following theorem which provides a partial positive answer to Question 1 (and to [7, Question 381] as well), as well as some generalizations of results of $[1,3]$.

THEOREM 1.1. A second countable space $X$ is weakly orderable if and only if it has a continuous weak selection.

It should be remarked that if $X$ is a second countable totally disconnected space, then one can easily construct a continuous injective map of $X$ into the Cantor set $\mathfrak{C}$ (see Remarks 5.5 and 5.6). Consequently, every second countable totally disconnected space is weakly orderable. According to $[10$, Lemma 7.5.1], this also implies that, in this case, the collection $\mathscr{C}(X)$ of all nonempty compact subsets of $X$ has a continuous selection. This settles [8, Question 5] and [7, Question 395].

The proof of Theorem 1.1 in the general case is based on Purisch's technique [12] that deals with orderability and suborderability of metrizable spaces (see Sections 3 and 4). To prepare for this, in Section 2 we provide several facts about continuous weak selections and connected components. The proof of Theorem 1.1 will be finally accomplished in Section 5. The technique developed to achieve that proof also allows us to obtain some further natural generalizations of Theorem 1.1 (see Section 5). For instance, we demonstrate that every separable space $X$ with a continuous weak selection must be weakly orderable provided the set $\left\{S \in \mathscr{F}_{2}(X):|S|=2\right\}$ is collectionwise Hausdorff in the Vietoris topology (Theorem 5.4).

2. Continuous weak selections and components. A subset $\mathscr{E} \subset X^{2}$ is usually called a binary relation on $X$, or just a relation on $X$, and we write $x \mathscr{E} y$ instead of $(x, y) \in \mathscr{E}$. A relation $\mathscr{E} \subset X^{2}$ is called a selection relation [6] if $\mathscr{E}$ is total and antisymmetric. A selection relation is a linear order if and only if it is transitive. 
Note that $\preceq_{f}$ is a selection relation on $X$ whenever $f$ is a weak selection for $X$. The converse is also true. Namely, let $\mathscr{E} \subset X^{2}$ be a selection relation, and for $x, y \in X$ define $f_{\mathscr{E}}(\{x, y\})=x$ if $x \mathscr{E} y$. Then $f_{\mathscr{E}}$ is a weak selection for $X$. Thus, there is a one-to-one correspondence between the weak selections for $X$ and the selection relations on $X$.

For a space $X$, a selection relation $\mathscr{E} \subset X^{2}$ and $x \in X$, define the following $\mathscr{E}$-open intervals:

$$
\begin{aligned}
(\leftarrow, x)_{\mathscr{E}} & =\{y \in X: y \neq x \text { and } y \mathscr{E} x\} \\
(x, \rightarrow)_{\mathscr{E}} & =\{y \in X: y \neq x \text { and } x \mathscr{E} y\}
\end{aligned}
$$

In the same way, we define the corresponding $\mathscr{E}$-closed intervals:

$$
(\leftarrow, x]_{\mathscr{E}}=\{y \in X: y \mathscr{E} x\} \quad \text { and } \quad[x, \rightarrow)_{\mathscr{E}}=\{y \in X: x \mathscr{E} y\} .
$$

Finally, for $x, y \in X$, we will also use the following composite $\mathscr{E}$-intervals:

$$
(x, y)_{\mathscr{E}}=(x, \rightarrow)_{\mathscr{E}} \cap(\leftarrow, y)_{\mathscr{E}} \quad \text { and } \quad[x, y]_{\mathscr{E}}=[x, \rightarrow)_{\mathscr{E}} \cap(\leftarrow, y]_{\mathscr{E}} .
$$

Since $\mathscr{E}$ is not necessarily transitive, both $(x, y)_{\mathscr{E}}$ and $(y, x)_{\mathscr{E}}$ may be nonempty, and similarly for $[x, y]_{\mathscr{E}}$ and $[y, x]_{\mathscr{E}}$.

The following two observations are due to Michael [10] (see also Eilenberg [2]).

Proposition 2.1 ([10]). If $f$ is a continuous weak selection for $X$, then all $\preceq_{f}$-open intervals $\left(\leftarrow, x \varliminf_{f}\right.$ and $\left(x, \longrightarrow_{\varliminf_{f}}, x \in X\right.$, are open in $X$. In particular, all $\preceq_{f}$-closed intervals $(\leftarrow, x]_{f}$ and $\left[x, \rightarrow_{\preceq_{f}}, x \in X\right.$, are closed in $X$.

TheOREM $2.2([2,10])$. If $C$ is a connected space and $f$ is a continuous weak selection for $C$, then $\preceq_{f}$ is a linear order on $C$. Also, there exists exactly one other continuous weak selection for $C$.

To any selection $f: \mathscr{F}_{2}(X) \rightarrow X$ one can associate another one $f^{\perp}$ : $\mathscr{F}_{2}(X) \rightarrow X$ defined by $S=\left\{f(S), f^{\perp}(S)\right\}, S \in \mathscr{F}_{2}(X)$. Note that if

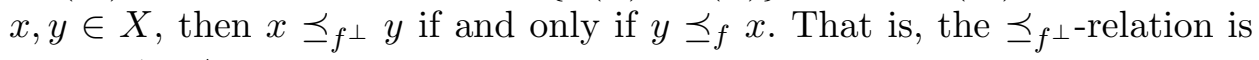
reverse to $\preceq_{f}$.

In what follows, for convenience, we let $\mathbb{W}_{\mathrm{cs}}(X)$ be the set of all continuous weak selections for a space $X$. It is well-known that $f \in \mathbb{W}_{\mathrm{cs}}(X)$ if and only if $f^{\perp} \in \mathbb{W}_{\text {cs }}(X)$ (see, for instance, [4, Theorem 3.5]). This implies the following simple observation, which will be found useful.

Proposition 2.3. Let $X$ be a space with $\mathbb{W}_{\mathrm{cs}}(X) \neq \emptyset$, and let $x, y \in X$. Then there exist $f, g \in \mathbb{W}_{\mathrm{cs}}(X)$ such that $x \preceq_{f} y$ and $y \preceq_{g} x$.

For a space $X$ and $x \in X$, we will use $\mathscr{C}[x]$ to denote the component of the point $x$, and $\mathscr{C}^{*}[x]$ for the corresponding quasi-component. Recall that 


$$
\begin{aligned}
\mathscr{C}[x] & =\bigcup\{C \subset X: x \in C \text { and } C \text { is connected }\}, \\
\mathscr{C}^{*}[x] & =\bigcap\{C \subset X: x \in C \text { and } C \text { is clopen }\} .
\end{aligned}
$$

TheOREM 2.4 ([5]). If $X$ has a continuous weak selection, then $\mathscr{C}[x]=$ $\mathscr{C}^{*}[x]$ for every $x \in X$.

Here are two other properties relating to connected subsets of such spaces.

LEMMA 2.5. If $f$ is a continuous weak selection for $X, C$ is a connected subset of $X$, and $x, y \in C$ with $x \prec_{f} y$, then

(a) $\emptyset \neq(x, y)_{f} \subset C$.

(b) $[x, y]_{f}$ is a connected subset of $X$.

Proof. The inclusion $(x, y)_{f} \subset C$ follows by [5, Lemma 3.3]. Suppose that $(x, y) \preceq_{f}=\emptyset$. Then, by Proposition $2.1, A=(\leftarrow, y) \preceq_{f} \cap C=(\leftarrow, x]_{\preceq_{f}} \cap C$ is a clopen subset of $C$ such that $x \in A$ and $y \notin A$, a contradiction, which demonstrates (a).

To show (b), we follow the proof of Theorem 2.4 in [5]. Suppose that there exists a clopen (in $[x, y]_{\preceq_{f}}$ ) neighbourhood $W \subset[x, y]_{\preceq_{f}}$ of $y$ such that $[x, y] \preceq_{f} \backslash W \neq \emptyset$. Choose a point $z \in[x, y]_{\preceq_{f}} \backslash W$, and set $T=W \cap[z, y]_{\preceq_{f}}$. Thus, we get a clopen (in $[z, y]_{\preceq_{f}}$ ) neighbourhood $T$ of $y$, with $z \notin T$. Then the set $G=T \cup\left[y, \rightarrow \varliminf_{f}\right.$ is clopen in $X$. Indeed, by Proposition 2.1, $G$ is closed as a union of two closed sets. Since $T \subset\left(z, \longrightarrow_{f} \preceq_{f}\right.$, there exists an open subset $E \subset\left(z, \rightarrow \preceq_{f}\right.$ with $E \cap[z, y]_{\preceq_{f}}=T$. Hence, by Proposition 2.1, $G=E \cup(y, \rightarrow) \preceq_{f}$ is also open in $X$. However, this is impossible because, by (a), $z \in[x, y]_{f} \backslash G \subset C \backslash G$ and $y \in C \cap G$.

Proposition 2.6. If $f$ is a continuous weak selection for $X, C$ is a connected subset of $X$, and $y \in X \backslash C$, then $C \subset(\leftarrow, y) \preceq_{f}$ or $C \subset\left(y, \longrightarrow_{\varliminf_{f}}\right.$.

Proof. Since $y \notin C$, we have

$$
(\leftarrow, y) \preceq_{f} \cap C=\left(\leftarrow , y \underline { \preceq } _ { f } \cap C \quad \text { and } \quad \left(y, \longrightarrow_{\preceq_{f}} \cap C=\left[y, \longrightarrow_{\preceq_{f}} \cap C .\right.\right.\right.
$$

Hence, by Proposition $2.1,(\leftarrow, y) \preceq_{f} \cap C$ and $\left(y, \longrightarrow_{\beth_{f}} \cap C\right.$ are disjoint clopen subsets of $C$. Since $C \subset X \backslash\{y\}=(\leftarrow, y) \preceq_{f} \cup(y, \rightarrow) \preceq_{f}$, the proof is complete.

A point $p$ of a connected space $C$ is called a cut point if $C \backslash\{p\}$ is not connected, and a noncut point otherwise. We let $\operatorname{ct}(C)$ be the set of all cut points of $C$, and $\operatorname{nct}(C)$ be the set of all noncut points.

COROllary 2.7. Let $f$ be a continuous weak selection for $X, C$ be a connected subset of $X$, and let $p \in C$. Then:

(a) $p \in \operatorname{nct}(C)$ if and only if $x \preceq_{f} p$ for every $x \in C$ or $p \preceq_{f} x$ for every $x \in C$. 
(b) $p \in \operatorname{ct}(C)$ if and only if there are $s, t \in C$ with $s \prec_{f} p \prec_{f} t$. In particular, $|\operatorname{nct}(C)| \leq 2$ and $\operatorname{ct}(C)$ is open in $X$.

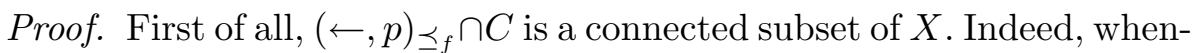
ever $(\leftarrow, p)_{\preceq_{f}} \cap C \neq \emptyset$, pick a point $c \in(\leftarrow, p)_{\beth_{f}} \cap C$. Then the statement follows by Theorem 2.2 and Lemma 2.5 because

$$
(\leftarrow, p)_{f} \cap C=\bigcup\left\{[x, y]_{f}: x, y \in C \text { and } x \preceq_{f} c \preceq_{f} y \prec_{f} p\right\} .
$$

In the same way, $(p, \rightarrow)_{f} \cap C$ is a connected subset of $X$. On the other hand, by Proposition 2.6, $C \backslash\{p\} \subset(\leftarrow, p)_{\preceq_{f}}$ or $C \backslash\{p\} \subset\left(p, \rightarrow_{\preceq_{f}}\right.$ provided $C \backslash\{p\}$ is connected. Consequently, $C \backslash\{p\}$ is connected if and only if $C \backslash\{p\}=(\leftarrow, p)_{\preceq_{f}} \cap C$ or $C \backslash\{p\}=(p, \rightarrow)_{\beth_{f}} \cap C$, which is (a). Since (b) follows from (a), the proof is complete.

3. Purisch sets. Relying on a construction in [12], to every space $X$ with $\mathbb{W}_{\mathrm{cs}}(X) \neq \emptyset$, we are going to associate a totally disconnected subset $Z \subset X$ which preserves the information on the components of $X$.

Definition 3.1 ([12]). Let $X$ be a space such that $|\operatorname{nct}(\mathscr{C}[x])| \leq 2$ for every $x \in X$. We shall say that a subset $Z \subset X$ is a Purisch set if for every $x \in X$ the following holds:

(a) $\mathscr{C}[x] \subset Z$ provided $\mathscr{C}[x]$ is a singleton.

(b) $|\mathscr{C}[x] \cap Z|=1$ provided $\operatorname{nct}(\mathscr{C}[x])=\emptyset$.

(c) $|\mathscr{C}[x] \cap Z|=2$ and $\operatorname{nct}(\mathscr{C}[x]) \subset Z$ otherwise.

Below we summarize some basic properties of Purisch sets. Some of them are not directly related to the proof of Theorem 1.1, but they show that Purisch sets are not as arbitrary as it might seem at first.

Proposition 3.2. Let $X$ be a space with $\mathbb{W}_{\mathrm{cs}}(X) \neq \emptyset$. Then $X$ has at least one Purisch subset, and any Purisch subset of $X$ is totally disconnected.

Proof. According to Corollary 2.7 and Definition 3.1, $X$ has at least one Purisch subset. Let $Z$ be any Purisch subset $X$. On the one hand, the component of each point of $Z$ is contained in the corresponding component of that point in $X$. Consequently, by Definition 3.1, the components (in $Z$ ) of the points of $Z$ must be singletons. On the other hand, $\mathbb{W}_{\mathrm{cs}}(Z) \neq \emptyset$ because $\mathbb{W}_{\mathrm{cs}}(X) \neq \emptyset$. Hence, by Theorem 2.4, $Z$ must be totally disconnected.

Proposition 3.3. Let $X$ be a space with $\mathbb{W}_{\mathrm{cs}}(X) \neq \emptyset$, and let $Z \subset X$ be a Purisch subset. Then $Z$ is closed in $X$.

Proof. Fix $x \in X \backslash Z$. Then, by Definition 3.1, $x \in \operatorname{ct}(\mathscr{C}[x])$. Since $|\mathscr{C}[x] \cap Z| \leq 2$, by Corollary $2.7, U=\operatorname{ct}(\mathscr{C}[x]) \backslash Z$ is a neighbourhood of $x$ in $X$. 
Suppose that $Z \subset X$ is a Purisch set. Following [12], for every $z \in Z$ we define a subset $\mathrm{nb}(z) \subset Z$ by setting $\mathrm{nb}(z)=\mathscr{C}[z] \cap Z$. The elements of $\mathrm{nb}(z)$ will be called neighbours of $z$. Clearly, $y \in \mathrm{nb}(z)$ if and only if $\mathrm{nb}(y)=\mathrm{nb}(z)$. Of course, $|\operatorname{nb}(z)| \leq 2$ for every $z \in Z$.

Definition 3.4. A clopen subset $W$ of a Purisch set $Z \subset X$ will be called order-regular if

(a) $|\{z \in W: \operatorname{nb}(z) \backslash W \neq \emptyset\}| \leq 1$,

(b) $W=U \cap Z$ for some open $U \subset X$ with $U \subset \bigcup\{\mathscr{C}[z]: z \in W\}$.

Let $\mathscr{O}_{\mathrm{r}}(Z)$ be the set of all order-regular subsets of $Z$.

Proposition 3.5. Let $X$ be a space with $\mathbb{W}_{\mathrm{cs}}(X) \neq \emptyset, Z \subset X$ be a Purisch set, and let $U \subset X$ be clopen. Then $U \cap Z \in \mathscr{O}_{\mathrm{r}}(Z)$.

Proof. Follows from the fact that $\mathscr{C}[z] \subset U$ for every $z \in U$.

Proposition 3.6. Let $X$ be a space, $f \in \mathbb{W}_{\mathrm{cs}}(X), Z \subset X$ be a Purisch set, and let $x \in X \backslash Z$. Then $(\leftarrow, x)_{\varliminf_{f}} \cap Z,\left(x, \rightarrow_{\varliminf_{f}} \cap Z \in \mathscr{O}_{\mathrm{r}}(Z)\right.$.

Proof. Fix $z \in\left(\leftarrow, x{\preceq_{f}}_{f} \cap Z\right.$ with $z \notin \mathscr{C}[x]$. Then $z \prec_{f} x$ and, by Proposition 2.6, $\mathrm{nb}(z) \subset \mathscr{C}[z] \subset(\leftarrow, x) \preceq_{f}$. Since the other case is symmetric, the proof is complete.

Proposition 3.7. Let $X$ be a space with $\mathbb{W}_{\mathrm{cS}}(X) \neq \emptyset, Z \subset X$ be a Purisch set, and let $V, W \in \mathscr{O}_{\mathrm{r}}(Z)$. Then there exists a finite pairwise disjoint family $\mathscr{U} \subset \mathscr{O}_{\mathrm{r}}(Z)$ such that $V \cap W=\bigcup \mathscr{U}$.

Proof. First of all, let us show that $V \cap W=O \cap Z$ for some open $O \subset X$ with $O \subset \bigcup\{\mathscr{C}[z]: z \in V \cap W\}$. By hypothesis, there are open (in $X$ ) subsets $O_{V} \subset \bigcup\{\mathscr{C}[z]: z \in V\}$ and $O_{W} \subset \bigcup\{\mathscr{C}[z]: z \in W\}$ such that $V=O_{V} \cap Z$ and $W=O_{W} \cap Z$. If $O_{V} \cap O_{W} \subset \bigcup\{\mathscr{C}[z]: z \in V \cap W\}$, set $O=O_{V} \cap O_{W}$. If there is a point $x \in\left(O_{V} \cap O_{W}\right) \backslash \bigcup\{\mathscr{C}[z]: z \in V \cap W\}$, then $x \in \mathscr{C}\left[z_{V}\right] \cap \mathscr{C}\left[z_{W}\right]$ for some $z_{V} \in V$ and $z_{W} \in W$ such that $z_{V} \notin W$ and $z_{W} \notin V$. In particular, $z_{V}$ and $z_{W}$ must be neighbours and, according to Definition 3.4, there could only be one pair of such neighbours. Consequently, $\left(O_{V} \cap O_{W}\right) \backslash \bigcup\{\mathscr{C}[z]: z \in V \cap W\} \subset \mathscr{C}[x]$ and, in this case, we can set $O=\left(O_{V} \cap O_{W}\right) \backslash \mathscr{C}[x]$.

Now, suppose $V \cap W \notin \mathscr{O}_{\mathrm{r}}(Z)$. Then there are distinct points $y, z \in V \cap W$ such that $\mathrm{nb}(y) \backslash V \neq \emptyset$ and $\mathrm{nb}(z) \backslash W \neq \emptyset$. In this case, $y$ and $z$ cannot be neighbours. Hence, $z \notin \mathscr{C}[y]$ and, by Theorem 2.4 , there is a clopen set $U \subset X$ such that $y \in U$ and $z \notin U$. Set $\mathscr{U}=\{V \cap W \cap U,(V \cap W) \backslash U\}$. To see that this works, fix a point $x \in V \cap W$ with $y \neq x \neq z$. Then $\mathrm{nb}(x) \subset V \cap W$, while $\mathscr{C}[x] \subset U$ or $\mathscr{C}[x] \subset X \backslash U$. Hence, $\mathrm{nb}(x) \subset V \cap W \cap U$ or $\mathrm{nb}(x) \subset(V \cap W) \backslash U$. 
4. Weak orderability and Purisch sets. We say that a sequence $\left\{\mathscr{U}_{n}: n<\omega\right\}$ of covers $\mathscr{U}_{n}, n<\omega$, of a space $Z$ is separating for the points of $Z$ if for any two distinct points $y, z \in Z$ there exists an $n<\omega$ such that $z \notin \bigcup\left\{U \in \mathscr{U}_{n}: y \in U\right\}$.

In this section, we prove a criterion of weak orderability which is actually inspired by the characterization of orderable and suborderable metrizable spaces in [12]. To this end, we adopt some of the terminology in [12]. Suppose that $X$ is a space with $\mathbb{W}_{\mathrm{cs}}(X) \neq \emptyset$, and $Z \subset X$ is a Purisch set. A linear order $\leq$ on a pairwise disjoint cover $\mathscr{W} \subset \mathscr{O}_{\mathrm{r}}(Z)$ of $Z$ is a $\mathscr{W}$-ordering [12] if two distinct members of $\mathscr{W}$ which contain neighbours have no other member of $\mathscr{W}$ between them with respect to $\leq$. As usual, if $\leq$ is a linear order on $\mathscr{W}$, we write $W<W^{\prime}$ if $W, W^{\prime} \in \mathscr{W}$ are distinct and $W \leq W^{\prime}$.

If $\leq$ is a $\mathscr{W}$-ordering on a pairwise disjoint cover $\mathscr{W} \subset \mathscr{O}_{\mathrm{r}}(Z)$ of $Z$, then there exists a unique map

$$
f=(g, h): \mathscr{W} \rightarrow(\{Z\} \times\{Z\}) \cup(\{Z\} \times Z) \cup(Z \times\{Z\}),
$$

called the $(\mathscr{W}, \leq)$-map $[12]$, which is defined in the following way:

(i) if $W \in \mathscr{W}$ and $\mathrm{nb}(z) \subset W$ for every $z \in W$, then $f(W)=(Z, Z)$,

(ii) if $y, y^{\prime} \in Z$ are neighbours and $W, W^{\prime} \in \mathscr{W}$ are such that $y \in W$, $y^{\prime} \in W^{\prime}$ and $W<W^{\prime}$, then $f(W)=(Z, y)$ and $f\left(W^{\prime}\right)=\left(y^{\prime}, Z\right)$.

Let $\mathscr{W} \subset \mathscr{O}_{\mathrm{r}}(Z)$ be a pairwise disjoint cover of $Z, \leq_{\mathscr{W}}$ be a $\mathscr{W}$-ordering on $\mathscr{W}$, and let $f_{\mathscr{W}}=\left(g_{\mathscr{W}}, h_{\mathscr{W}}\right)$ be the corresponding $(\mathscr{W}, \leq \mathscr{W})$-map. Also, let $\mathscr{V} \subset \mathscr{O}_{\mathrm{r}}(Z)$ be another pairwise disjoint cover of $Z$ which is a refinement of $\mathscr{W}$. Whenever $W \in \mathscr{W}$, set $\mathscr{V}(W)=\{V \in \mathscr{V}: V \subset W\}$. A $\mathscr{V}$-ordering $\leq_{\mathscr{V}}$ on $\mathscr{V}$ will be called $\leq_{\mathscr{W}}$-compatible if

(i) $V_{1}<\mathscr{V} V_{2}$ provided $V_{i} \in \mathscr{V}\left(W_{i}\right), i=1,2$, for some $W_{1}, W_{2} \in \mathscr{W}$ with $W_{1}<\mathscr{W} W_{2}$.

(ii) If $g_{\mathscr{W}}(W) \neq Z$ for some $W \in \mathscr{W}$ and $V \in \mathscr{V}(W)$ contains $g_{\mathscr{W}}(W)$, then $V$ is the first element of $\mathscr{V}(W)$ with respect to $\leq_{\mathscr{V}}$.

(iii) If $h_{\mathscr{W}}(W) \neq Z$ for some $W \in \mathscr{W}$ and $V \in \mathscr{V}(W)$ contains $h_{\mathscr{W}}(W)$, then $V$ is the last element of $\mathscr{V}(W)$ with respect to $\leq_{\mathscr{V}}$.

Finally, recall that a subset $A \subset Z$ of a linearly ordered set $(Z, \leq)$ is called $\leq$-convex (or just convex) if $[y, z] \leq A$ for all $y, z \in A$ with $y \leq z$.

TheOREM 4.1. Let $X$ be a space with $\mathbb{W}_{\mathrm{cS}}(X) \neq \emptyset$, and let $Z \subset X$ be a Purisch set. Suppose that $\mathscr{W}_{n} \subset \mathscr{O}_{\mathrm{r}}(Z), n<\omega$, are pairwise disjoint covers of $Z$ such that

(a) $\mathscr{W}_{n+1}$ is a refinement of $\mathscr{W}_{n}$ for every $n<\omega$,

(b) the sequence $\left\{\mathscr{W}_{n}: n<\omega\right\}$ is separating for the points of $Z$.

Then $X$ is weakly orderable. 
Proof. Just as in the proof of [12, Lemma 3.2], using (a), for every $n<\omega$ one can define a $\mathscr{W}_{n}$-ordering $\leq_{n}$ on $\mathscr{W}_{n}$ such that

$$
\text { the } \mathscr{W}_{n+1} \text {-ordering } \leq_{n+1} \text { is } \leq_{n} \text {-compatible, } n<\omega \text {. }
$$

Also, for every $n<\omega$, let $f_{n}=\left(g_{n}, h_{n}\right)$ be the corresponding $\left(\mathscr{W}_{n}, \leq_{n}\right)$-map. Finally, define a relation $\leq$ on $Z$ by writing $y \leq z$ if $y=z$ or there exists an $n<\omega$ and members $V, W \in \mathscr{W}_{n}$ such that $y \in V, z \in W$ and $V<_{n} W$. According to (4.1), the relation $\leq$ is well-defined and, in fact, by (a), (b) and (4.1), it is a linear order on $Z$.

In what follows, let $\mathscr{N}(Z)=\{\mathrm{nb}(z): z \in Z\}$. Then $\nu=\mathscr{C}[z] \cap Z$ for every $\nu \in \mathscr{N}(Z)$ and $z \in \nu$. Observe that

$$
\nu \text { is } \leq \text {-convex for every } \nu \in \mathscr{N}(Z) \text {. }
$$

Indeed, if $\nu$ is a singleton, this is obvious. Suppose that $y, z \in \nu$ and $y<z$. If $x \in Z \backslash \nu$, then, by (a) and (b), there exists an $n<\omega$ and distinct $U, V, W \in \mathscr{W}_{n}$ such that $x \in U, y \in V$ and $z \in W$. Since $\leq_{n}$ is a $\mathscr{W}_{n^{-}}$ ordering, we find that $U<_{n} V$ or $W<_{n} U$ because $V<_{n} W$. Consequently, $x \notin[y, z]_{\leq}$.

Finally, we are going to define a linear order $\preceq$ on $X$ generated by the $\leq$-order on $Z$. To this end, by Proposition 2.3, for every $\nu \in \mathscr{N}(Z)$ there exists a selection $f_{\nu} \in \mathbb{W}_{\mathrm{cS}}(X)$ such that, whenever $y, z \in \nu$,

$$
y \preceq f_{\nu} z \text { if and only if } y \leq z .
$$

For $s, t \in X$, let $\nu(s)=\mathscr{C}[s] \cap Z$ and define $s \preceq t$ if $\nu=\nu(s)=\nu(t)$ and $s \preceq f_{\nu} t$, or $\nu(s) \neq \nu(t)$ and $y \leq z$ for some (every) $y \in \nu(s)$ and $z \in \nu(t)$. In other words, the relation $\preceq$ restricted to the component $\mathscr{C}[x]$ of a point $x \in X$ is the selection relation $\preceq_{f_{\nu}}$ corresponding to $\nu=\mathscr{C}[x] \cap Z$, while between distinct components of $X$ it is the linear order $\leq$ on $Z$. Since $\leq$ is a linear order on $Z$ and, by Theorem $2.2, \preceq_{f_{\nu}}, \nu \in \mathscr{N}(Z)$, is a linear order on each component of $X,(4.2)$ and (4.3) imply that $\preceq$ is a linear order on $X$. So, it only remains to show that $X$ is weakly orderable with respect to this order. Fix $x \in X$ and $y \in(x, \rightarrow)_{\preceq}$, and let $\nu=\mathscr{C}[y] \cap Z$. We have the following possibilities:

$\left(\mathbf{W O}_{\mathbf{1}}\right) y$ is a cut point of $\mathscr{C}[y]$. In this case, there are $s, t \in \mathscr{C}[y]$ such that $x \prec s \prec_{f_{\nu}} y \prec_{f_{\nu}} t$. Indeed, if $x \notin \mathscr{C}[y]$, then, by the definition of $\preceq$, we have $x \prec z$ for every $z \in \mathscr{C}[y]$, hence Corollary 2.7 implies the statement. If $x \in \mathscr{C}[y]$, then $x \prec_{f_{\nu}} y$, and the existence of $s, t$ follows from Lemma 2.5 and Corollary 2.7. Thus, in this case, $\left(s, t{\varliminf_{f \nu}} \subset \mathscr{C}[y]\right.$ by Lemma $2.5 ;(s, t)_{f_{\nu}}$ is open in $X$ by Propositions 2.1; and $y \in(s, t)_{\preceq_{f_{\nu}}} \subset(x, \rightarrow)_{\preceq}$ by the definition of $\preceq$.

$\left(\mathbf{W O}_{2}\right) y$ is a noncut point of $\mathscr{C}[y]$ and $x \in \mathscr{C}[y]$. By Definition 3.1, $y$ has a neighbour $z$ in $Z$, i.e. there exists a point $z \in \nu$ with $z \neq y$. By 
(4.3) and Corollary 2.7, this implies that $z<y$ because $x \prec f_{\nu} y$. Now, on the one hand, there exist $n<\omega$ and $W(z), W(y) \in \mathscr{W}_{n}$ such that $z \in W(z)$, $y \in W(y)$ and $W(z)<_{n} W(y)$. On the other hand, by Definition 3.4, there also exists an open (in $X$ ) subset $U(y) \subset \bigcup\{\mathscr{C}[s]: s \in W(y)\}$ with $U(y) \cap Z=W(y)$. Then, by Proposition 2.1, $O(y)=U(y) \cap(x, \rightarrow) \preceq_{f_{\nu}}$ is a neighbourhood of $y$ in $X$. We now show that $O(y) \subset(x, \rightarrow)_{\preceq}$. Fix $t \in O(y)$. If $t \in \mathscr{C}[y]$, then $x \prec t$ because $x \prec f_{\nu}$. If $t \notin \mathscr{C}[y]$, then there exists an $s \in \mathscr{C}[t] \cap W(y)$ because $U(y) \subset \bigcup\{\mathscr{C}[s]: s \in W(y)\}$. In this case, observe that, by $(4.1), g_{k}(W)=y$ for every $k \geq n$ and $W \in \mathscr{W}_{k}$, with $y \in W$. Then, by (a) and (b), there exist $m \geq n, W \in \mathscr{W}_{m}$ and $V(y) \in \mathscr{W}_{m+1}(W)$ such that $y, s \in W, y \in V(y)$, but $s \notin V(y)$. According to (4.1) once again, $V(y)$ is the first element of $\mathscr{W}_{m+1}(W)$ with respect to $\leq_{m+1}$ because $g_{m}(W)=y$. Hence, $y<s$, and therefore, by the definition of $\preceq, x \prec y \prec t$ because $t \in \mathscr{C}[s]$.

$\left(\mathbf{W O}_{3}\right) y$ is a noncut point of $\mathscr{C}[y]$ and $x \notin \mathscr{C}[y]$. Fix $z \in \mathscr{C}[x] \cap Z$. Since $x \prec y$, we have $z<y$. Hence, there exist $n<\omega$ and $W(z), W(y) \in \mathscr{W}_{n}$ such that $z \in W(z), y \in W(y)$ and $W(z)<_{n} W(y)$. Let $U(y)$ be an open (in $X$ ) subset of $\bigcup\{\mathscr{C}[s]: s \in W(y)\}$ with $U(y) \cap Z=W(y)$. On the other hand, by Theorem 2.4, there exists a clopen subset $V$ of $X$ such that $x \notin V$ and $\mathscr{C}[y] \subset V$. We now show that $O(y)=V \cap U(y) \subset(x, \rightarrow)_{\preceq}$. Fix $t \in O(y)$. Then there exists an $s \in W(y)$ such that $t \in \mathscr{C}[s]$. Since $t \in V$, this implies that $\mathscr{C}[s] \subset V$. Hence, by the definition of $\preceq$, we have $x \prec t$ because $z<s$, $x \in \mathscr{C}[z], t \in \mathscr{C}[s]$ and $\mathscr{C}[z] \cap \mathscr{C}[s]=\emptyset$.

This completes the verification that $(x, \rightarrow)_{\preceq}$ is open in $X$. The proof that $(\leftarrow, x)_{\preceq}$ is open in $X$ is completely analogous.

5. Separating points in Purisch sets. In this section, we finish the proof of Theorem 1.1. To prepare for this, we need the following consequence of Theorem 4.1.

Corollary 5.1. Let $X$ be a space with $\mathbb{W}_{\mathrm{cs}}(X) \neq \emptyset, Z \subset X$ be a Purisch set, and let $\mathscr{U}_{n} \subset \mathscr{O}_{\mathrm{r}}(Z), n<\omega$, be pairwise disjoint covers of $Z$ such that the sequence $\left\{\mathscr{U}_{n}: n<\omega\right\}$ is separating for the points of $Z$. Then $X$ is weakly orderable.

Proof. According to Theorem 4.1, it suffices to show that $Z$ has a sequence of pairwise disjoint covers $\mathscr{W}_{n} \subset \mathscr{O}_{\mathrm{r}}(Z), n<\omega$, satisfying (a) and (b) of that theorem. To this end, set $\mathscr{W}_{0}=\mathscr{U}_{0}$, and proceed by induction. Suppose that $\mathscr{W}_{n} \subset \mathscr{O}_{\mathrm{r}}(Z)$ is a pairwise disjoint cover of $Z$ for some $n<\omega$. Then, by Proposition 3.7, for any $W \in \mathscr{W}_{n}$ and $U \in \mathscr{U}_{n+1}$, there exists a finite pairwise disjoint family $\mathscr{W}_{n+1}(W, U) \subset \mathscr{O}_{\mathrm{r}}(Z)$ such that $W \cap U=\bigcup \mathscr{W}_{n+1}(W, U)$. 
Finally, set

$$
\mathscr{W}_{n+1}=\bigcup\left\{\mathscr{W}_{n+1}(W, U): W \in \mathscr{W}_{n} \text { and } U \in \mathscr{U}_{n+1}\right\} .
$$

The sequence $\left\{\mathscr{W}_{n}: n<\omega\right\}$ is as required.

In fact, we will mainly rely on the following special case of Corollary 5.1. In what follows, for a set $Z$, we let $[Z]^{2}=\{S \subset Z:|S|=2\}$.

Corollary 5.2. Let $X$ be a space with $\mathbb{W}_{\mathrm{cs}}(X) \neq \emptyset, Z \subset X$ be a Purisch set, and let $\mathscr{U}_{n} \subset \mathscr{O}_{\mathrm{r}}(Z), n<\omega$, be a sequence of 2-element disjoint covers of $Z$ such that $[Z]^{2}=\bigcup\left\{\left\langle\mathscr{U}_{n}\right\rangle: n<\omega\right\}$. Then $X$ is weakly orderable.

Proof. Fix $n<\omega$ and distinct $y, z \in Z$. Then $y$ and $z$ are in distinct members of $\mathscr{U}_{n}$ if and only if $\{y, z\} \in\left\langle\mathscr{U}_{n}\right\rangle$. Consequently, the hypothesis that $[Z]^{2}=\bigcup\left\{\left\langle\mathscr{U}_{n}\right\rangle: n<\omega\right\}$ implies that the sequence $\left\{\mathscr{U}_{n}: n<\omega\right\}$ is separating for the points of $Z$. Thus, Corollary 5.1 completes the proof.

For a space $Y$, we will use $\ell(Y)$ to denote the Lindelöf number of $Y$. Note that, by [10, Lemma 7.5.1], every weakly orderable space has a continuous weak selection. Hence, Theorem 1.1 is a consequence of the following more general result.

TheOrem 5.3. Let $X$ be a space with $\mathbb{W}_{\mathrm{cs}}(X) \neq \emptyset$ and $\ell\left([X]^{2}\right) \leq \omega$. Then $X$ is weakly orderable.

Proof. Choose a Purisch set $Z \subset X$. According to Corollary 5.2, it suffices to show that there exists a sequence $\mathscr{U}_{n} \subset \mathscr{O}_{\mathrm{r}}(Z), n<\omega$, of 2-element disjoint covers of $Z$ such that $[Z]^{2}=\bigcup\left\{\left\langle\mathscr{U}_{n}\right\rangle: n<\omega\right\}$. To this end, fix a selection $f \in \mathbb{W}_{\mathrm{cs}}(X)$ and an $\alpha=\{y, z\} \in[Z]^{2}$. We can assume that $y \prec_{f} z$. If $y$ and $z$ are not neighbours, then $\mathscr{C}[y] \cap \mathscr{C}[z]=\emptyset$. Hence, by Theorem 2.4, there exists a clopen subset $V$ of $X$ such that $\mathscr{C}[y] \subset V$ and $\mathscr{C}[z] \subset X \backslash V$. Then set $\mathscr{U}_{\alpha}=\{V \cap Z, Z \backslash V\}$, which is a 2-element disjoint cover of $Z$ such that $\alpha \in\left\langle\mathscr{U}_{\alpha}\right\rangle$ and, by Proposition 3.5, $\mathscr{U}_{\alpha} \subset \mathscr{O}_{\mathrm{r}}(Z)$. If $y$ and $z$ are neighbours, then $\mathscr{C}[y]=\mathscr{C}[z]$ and, by Lemma 2.5, there exists a point $x \in(y, z) \preceq_{f} \subset \mathscr{C}[y]$. Now, set $\mathscr{U}_{\alpha}=\left\{(\leftarrow, x)_{\preceq_{f}} \cap Z,\left(x, \longrightarrow_{\varliminf_{f}} \cap Z\right\}\right.$. Then $\alpha \in\left\langle\mathscr{U}_{\alpha}\right\rangle$, and the family $\mathscr{U}_{\alpha}$ is a 2-element disjoint cover of $Z$ because $x \notin Z$, while, by Proposition 3.6, $\mathscr{U}_{\alpha} \subset \mathscr{O}_{\mathrm{r}}(Z)$. Thus, we get an open cover $\left\{\mathscr{U}_{\alpha}: \alpha \in[Z]^{2}\right\}$ of $[Z]^{2}$. By Proposition 3.3, $Z$ is a closed

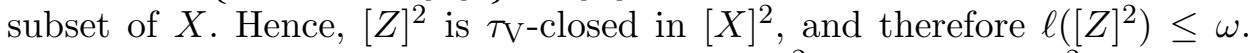
So, there exists a countable subset $\left\{\alpha(n) \in[Z]^{2}: n<\omega\right\} \subset[Z]^{2}$ such that $[Z]^{2}=\bigcup\left\{\left\langle\mathscr{U}_{\alpha(n)}\right\rangle: n<\omega\right\}$. Finally, let $\mathscr{U}_{n}=\mathscr{U}_{\alpha(n)}, n<\omega$, which completes the proof.

Motivated by Theorem 5.3, we have the following natural question.

Question 2. Let $X$ be a space such that $\ell\left(X^{2}\right) \leq \omega$ and $\mathbb{W}_{\mathrm{cs}}(X) \neq \emptyset$. Is then $X$ weakly orderable? 
The answer to Question 2 is "Yes" if $\Delta(X)=\{(x, x): x \in X\}$ is a $G_{\delta^{-}}$ set in $X^{2}$. In this case, $\ell\left(X^{2} \backslash \Delta(X)\right) \leq \omega$, which implies that $\ell\left([X]^{2}\right) \leq \omega$ because the map $h: X^{2} \backslash \Delta(X) \rightarrow[X]^{2}$, defined by $h(x, y)=\{x, y\}, x, y \in$ $X^{2} \backslash \Delta(X)$, is a continuous surjection.

We finish this paper by demonstrating the following further generalization of Theorem 1.1, in particular of [1, Theorem 2.2] as well.

TheOREm 5.4. Let $X$ be a separable space such that $[X]^{2}$ is collectionwise Hausdorff and $\mathbb{W}_{\mathrm{cs}}(X) \neq \emptyset$. Then $X$ is weakly orderable.

Proof. Choose a Purisch set $Z \subset X$. Just as in the previous proof, it suffices to show that there exists a countable family $\mathscr{U}_{\alpha} \subset \mathscr{O}_{\mathrm{r}}(Z), \alpha \in \mathscr{A}$, of two-element disjoint covers of $Z$ such that $[Z]^{2}=\bigcup\left\{\left\langle\mathscr{U}_{\alpha}\right\rangle: \alpha \in \mathscr{A}\right\}$. Pick a selection $f \in \mathbb{W}_{\mathrm{cs}}(X)$, a countable dense subset $D \subset X$, and a pair of distinct points $\gamma=\{s, t\} \in[D]^{2}$ with $s \prec_{f} t$. If $\mathscr{C}[s]=\mathscr{C}[t]$, then as in the proof of Theorem 5.3, there exists a point $x \in \mathscr{C}[s] \backslash Z$ with $x \in(s, t)_{\prec_{f}}$. In this case, set $U_{s}=(\leftarrow, x)_{\beth_{f}} \cap Z$ and $U_{t}=(x, \rightarrow)_{f} \cap Z$. If $\mathscr{C}[s] \neq \mathscr{C}[t]$, then again as in the proof of Theorem 5.3, choose a clopen set $V \subset X$ with $\mathscr{C}[s] \subset V$ and $V \cap \mathscr{C}[t]=\emptyset$, but now set $W=(\leftarrow, t)_{\preceq_{f}} \cap V$, which is clopen in $X$ because $W=(\leftarrow, t]_{\preceq_{f}} \cap V$. Finally, set $U_{s}=\left((\leftarrow, s)_{\preceq_{f}} \cup W\right) \cap Z$ and $U_{t}=Z \backslash U_{s}$. In each of these cases, the $U_{s}$ and $U_{t}$ so constructed form a clopen partition $\mathscr{U}_{\gamma}=\left\{U_{s}, U_{t}\right\}$ of $Z$ such that $\mathscr{U}_{\gamma} \subset \mathscr{O}_{\mathrm{r}}(Z)$ and

$$
\{y, z\} \in\left\langle\mathscr{U}_{\gamma}\right\rangle \quad \text { for all } y, z \in Z \text { with } y \preceq_{f} s \preceq_{f} z \text { and } t \preceq_{f} z .
$$

Let $D_{0}$ be the set of all isolated points of $D$ (hence, of $X$ as well). Whenever $s \in D_{0}$, let $\sigma=\{s\}$ be the corresponding singleton, and let $\mathscr{U}_{\sigma}=\left\{(\leftarrow, s]_{\unlhd_{f}} \cap Z,(s, \rightarrow)_{\beth_{f}} \cap Z\right\}$. Since $\sigma=\{s\}$ is a clopen set in $X$, by Proposition 2.1 , both $\preceq_{f}$-intervals $(\leftarrow, s]_{\preceq_{f}}$ and $\left(s, \rightarrow \underline{\preceq}_{f}\right.$ are clopen in $X$. Hence, by Proposition 3.5, $\mathscr{U}_{\sigma} \subset \mathscr{O}_{\mathrm{r}}(Z)$, and clearly it is a 2-element disjoint cover of $Z$. Finally, set $\mathscr{D}=[D]^{2} \cup\left\{\{s\}: s \in D_{0}\right\}$ and $\mathscr{F}=[Z]^{2} \backslash \bigcup\left\{\left\langle\mathscr{U}_{\delta}\right\rangle\right.$ : $\delta \in \mathscr{D}\}$. Then $\mathscr{F}$ is a closed discrete subset of $[X]^{2}$. To see this, let us show that

$$
(y, z) \preceq_{f}=\emptyset=(z, y) \preceq_{f} \quad \text { for every }\{y, z\} \in \mathscr{F} .
$$

The verification of (5.2) follows in part [1, Fact 5]. Fix a pair $\{y, z\} \in \mathscr{F}$. Since $\{y, z\} \notin\left\langle\mathscr{U}_{\gamma}\right\rangle$ for every $\gamma \in[D]^{2}$, by $(5.1)$, we now have $\left|(y, z) \preceq_{f} \cap D\right| \leq 1$. Consequently, if $s \in(y, z) \preceq_{f} \cap D$, then $\sigma=\{s\}=(y, z) \preceq_{f} \cap D$, and therefore $s$ must be an isolated point of $D$. In this case, by construction, $\{y, z\} \in$ $\left\langle\mathscr{U}_{\sigma}\right\rangle$, which is impossible. Hence, $(y, z)_{f}=\emptyset$, which, in fact, completes the verification of (5.2).

Choose now $\beta=\{y, z\} \in \mathscr{F}$, with $y \prec_{f} z$, and let

$$
\mathscr{O}_{\beta}=\left\{(\leftarrow, z) \varliminf_{f},(y, \rightarrow)_{f}\right\} .
$$


Then $\left\langle\mathscr{O}_{\beta}\right\rangle \cap \mathscr{F}=\{\beta\}$. Indeed, if $\gamma=\{s, t\} \in\left\langle\mathscr{O}_{\beta}\right\rangle \cap \mathscr{F}$, then, say, $s \in$ $(\leftarrow, z) \preceq_{f}$ and $t \in(y, \rightarrow) \preceq_{f}$. Therefore, $s \preceq_{f} y$ and $z \preceq_{f} t$ because, by (5.2), $(y, z)_{f}=\emptyset$. According to (5.2) once again, we also have $(s, t)_{f}=\emptyset$, which implies that $\beta=\{y, z\} \subset[s, t]_{\preceq_{f}}=\{s, t\}=\gamma$, so $\gamma=\beta$. Thus, $\mathscr{F}$ is discrete in $[X]^{2}$, while, by Proposition 3.3 , it is closed in $[X]^{2}$, being closed in $[Z]^{2}$. Hence, it must be countable, because $[X]^{2}$ is collectionwise Hausdorff and $X$ is separable. Finally, for every such $\beta=\{y, z\} \in \mathscr{F}$, let $\mathscr{U}_{\beta}=\left\{O \cap Z: O \in \mathscr{O}_{\beta}\right\}$, where $\mathcal{O}_{\beta}$ is defined as in (5.3). By Proposition 3.5, $\mathscr{U}_{\beta} \subset \mathscr{O}_{\mathrm{r}}(Z)$, while, by (5.2), $\mathscr{U}_{\beta}$ is a 2-element disjoint cover of $Z$, with $\beta \in\left\langle\mathscr{U}_{\beta}\right\rangle$, because $(y, z)_{f}=\emptyset=(z, y)_{\beth_{f}}$. Then $\mathscr{U}_{\alpha} \in \mathscr{O}_{\mathrm{r}}(Z), \alpha \in \mathscr{A}$, is as required, where $\mathscr{A}=\mathscr{F} \cup \mathscr{D}$.

The following question is motivated by Theorem 5.4.

Question 3. Let $X$ be a separable collectionwise Hausdorff space such that $\mathbb{W}_{\mathrm{cs}}(X) \neq \emptyset$. Is then $X$ weakly orderable?

REMARK 5.5. If $X$ is an infinite totally disconnected space and $\ell\left([X]^{2}\right)$ $\leq \omega$, then there exists an injective continuous map $h$ of $X$ into the Cantor set $\mathfrak{C}$. To see this, construct a sequence $\left\{\mathscr{U}_{n}: n<\omega\right\}$ of finite pairwise disjoint open covers of $X$ which is separating for the points of $X$. This can be done by following precisely the arguments of Theorem 5.3. Namely, whenever $\alpha=\{x, y\} \in[X]^{2}$, choose a clopen set $V \subset X$ with $x \in V$ and $y \notin V$, and set $\mathscr{U}_{\alpha}=\{V, X \backslash V\}$. Then, just as in Theorem 5.3, there exists a countable subset $\left\{\alpha(n) \in[X]^{2}: n<\omega\right\} \subset[X]^{2}$ such that $[X]^{2}=$ $\bigcup\left\{\left\langle\mathscr{U}_{\alpha(n)}\right\rangle: n<\omega\right\}$. The covers $\mathscr{U}_{n}=\mathscr{U}_{\alpha(n)}, n<\omega$, are as required. Now, endow each $\mathscr{U}_{n}, n<\omega$, with the discrete topology, and, for every $n<\omega$, define a map $h_{n}: X \rightarrow \mathscr{U}_{n}$ by letting $h_{n}(x)$ be the element of $\mathscr{U}_{n}$ with $x \in h_{n}(x)$. Then each $h_{n}, n<\omega$, is continuous, hence so is the diagonal map

$$
h=\Delta\left\{h_{n}: n<\omega\right\}: X \rightarrow \prod\left\{\mathscr{U}_{n}: n<\omega\right\} .
$$

On the one hand, $\prod\left\{\mathscr{U}_{n}: n<\omega\right\}$ is homeomorphic to the Cantor set $\mathfrak{C}$ because each $\mathscr{U}_{n}, n<\omega$, is a 2 -point space. On the other hand, $h$ is injective because the sequence $\left\{\mathscr{U}_{n}: n<\omega\right\}$ is separating for the points of $X$.

REMARK 5.6. Suppose that $X$ is a second countable totally disconnected space. Another very elegant construction of a countable family $\left\{U_{\alpha}: \alpha \in \mathscr{A}\right\}$ of clopen subsets of $X$, which is separating for the points of $X$, was suggested to the author by Jan van Mill. Namely, take a countable base $\mathscr{B}$ of $X$, and consider the set $\mathscr{A}$ of all pairs $(B, D)$ of members of $\mathscr{B}$ for which there exists a clopen set $V \subset X$ such that $B \subset V$ and $V \cap D=\emptyset$. Fix one such clopen set $U_{\alpha}$ per pair $\alpha \in \mathscr{A}$. Then $\left\{U_{\alpha}: \alpha \in \mathscr{A}\right\}$ is as required. Indeed, if $x, y \in X$ and $x \neq y$, then there exists a clopen set $V \subset X$ with $x \in V$ and $y \notin V$. Select $B, D \in \mathscr{B}$ such that $x \in B \subset V$ and $y \in D \subset X \backslash V$. 
Then $\alpha=(B, D) \in \mathscr{A}$, while the clopen set $U_{\alpha}$ corresponding to $\alpha$ has the property that $x \in U_{\alpha}$ and $y \in X \backslash U_{\alpha}$.

Acknowledgements. The author would like to express his gratitude to Professor Jan van Mill and Professor Tsugunori Nogura for several valuable remarks.

Addendum (August, 2007). At the time when this manuscript was in the process of being accepted for publication, Michael Hrušák and Iván Martínez-Ruiz announced that they answered Question 1 in the negative by constructing a separable, first countable locally compact space which admits a continuous weak selection but is not weakly orderable. Their manuscript [9] is in preparation.

\section{References}

[1] C. Costantini, Weak orderability of some spaces which admit a weak selection, Comment. Math. Univ. Carolin. 47 (2006), 609-615.

[2] S. Eilenberg, Ordered topological spaces, Amer. J. Math. 63 (1941), 39-45.

[3] S. García-Ferreira, V. Gutev, T. Nogura, M. Sanchis, and A. Tomita, Extreme selections for hyperspaces of topological spaces, Topology Appl. 122 (2002), 157-181.

[4] V. Gutev and T. Nogura, Selections and order-like relations, Appl. Gen. Topol. 2 (2001), 205-218.

[5] - Vietoris continuous selections and disconnectedness-like properties, Proc. Amer. Math. Soc. 129 (2001), 2809-2815.

[6] -, A topology generated by selections, Topology Appl. 153 (2005), 900-911.

[7] -, Selection problems for hyperspaces, in: Open Problems in Topology 2, E. Pearl (ed.), Elsevier, Amsterdam, 2007, 161-170.

[8] -, Selections and totally disconnected spaces, Topology Appl. (2007), to appear.

[9] M. Hrušák and I. Martínez-Ruiz, Selections and weak orderability, in preparation, 2007.

[10] E. Michael, Topologies on spaces of subsets, Trans. Amer. Math. Soc. 71 (1951), $152-182$.

[11] J. van Mill and E. Wattel, Selections and orderability, Proc. Amer. Math. Soc. 83 (1981), 601-605.

[12] S. Purisch, The orderability and suborderability of metrizable spaces, Trans. Amer. Math. Soc. 226 (1977), 59-76.

School of Mathematical Sciences

University of KwaZulu-Natal

Westville Campus

Private Bag X54001, Durban 4000, South Africa

E-mail: gutev@ukzn.ac.za

URL: maths.za.org 